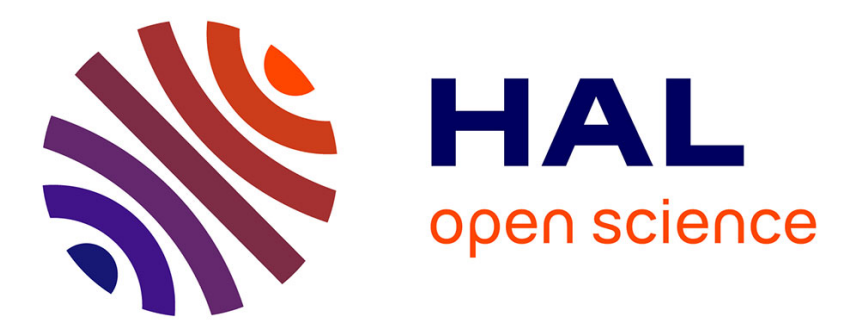

\title{
BOAR: Biprism based optical autocorrelation with retrieval
}

F. Billard, A. Dubrouil, E. Hertz, S Lecorné, E Szmygel, O. Faucher, P. Béjot

\section{To cite this version:}

F. Billard, A. Dubrouil, E. Hertz, S Lecorné, E Szmygel, et al.. BOAR: Biprism based optical autocorrelation with retrieval. Review of Scientific Instruments, 2019, 90 (6), 10.1063/1.5054357 . hal02389028

\section{HAL Id: hal-02389028 \\ https://hal.science/hal-02389028}

Submitted on 2 Dec 2019

HAL is a multi-disciplinary open access archive for the deposit and dissemination of scientific research documents, whether they are published or not. The documents may come from teaching and research institutions in France or abroad, or from public or private research centers.
L'archive ouverte pluridisciplinaire HAL, est destinée au dépôt et à la diffusion de documents scientifiques de niveau recherche, publiés ou non, émanant des établissements d'enseignement et de recherche français ou étrangers, des laboratoires publics ou privés. 


\section{BOAR: Biprism based optical autocorrelation with retrieval}

Cite as: Rev. Sci. Instrum. 90, 063110 (2019); https://doi.org/10.1063/1.5054357

Submitted: 31 August 2018 . Accepted: 03 June 2019 . Published Online: 25 June 2019

F. Billard (D), A. Dubrouil, E. Hertz, S. Lecorné, E. Szmygel, O. Faucher, and P. Béjot

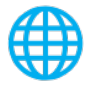

\section{ARTICLES YOU MAY BE INTERESTED IN}

\section{Autonomous sensor of electromagnetic field}

Review of Scientific Instruments 90, 064705 (2019); https://doi.org/10.1063/1.5090185

A direct comparison of high-speed methods for the numerical Abel transform

Review of Scientific Instruments 90, 065115 (2019); https://doi.org/10.1063/1.5092635

Cryogen-free variable temperature scanning SQUID microscope

Review of Scientific Instruments 90, 063705 (2019); https://doi.org/10.1063/1.5085008

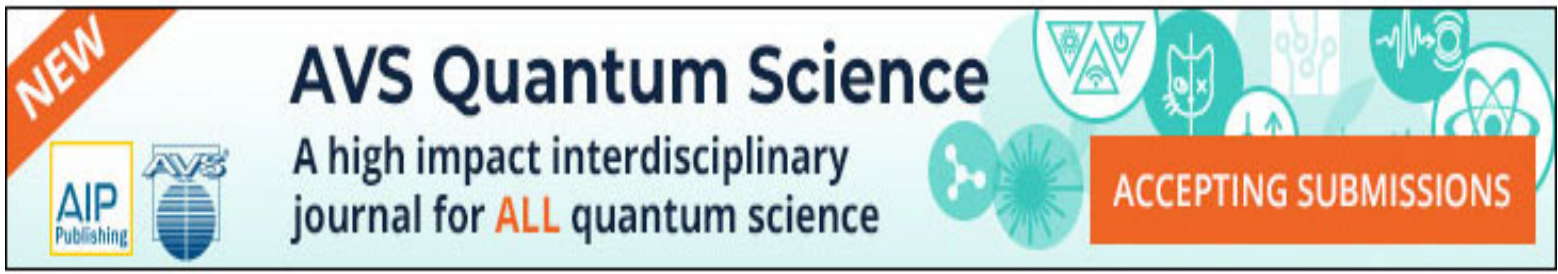




\title{
BOAR: Biprism based optical autocorrelation with retrieval
}

\author{
Cite as: Rev. Sci. Instrum. 90, 063110 (2019); doi: 10.1063/1.5054357 \\ Submitted: 31 August 2018 - Accepted: 3 June 2019 • \\ Published Online: 25 June 2019
}

\author{
F. Billard, ${ }^{\prime}$ (D) A. Dubrouil, ${ }^{2}$ E. Hertz, ${ }^{1}$ S. Lecorné, ${ }^{2}$ E. Szmygel, ${ }^{2}$ O. Faucher, ${ }^{1}$ and P. Béjot ${ }^{1, a)}$
}

\begin{abstract}
AFFILIATIONS
${ }^{1}$ Laboratoire Interdisciplinaire CARNOT de Bourgogne, UMR 6303 CNRS-Université Bourgogne Franche-Comté, 9 Ave. A. Savary, BP 47870, F-21078 Dijon Cedex, France

${ }^{2}$ Femto Easy, Bâtiment Sonora, Parc Scientifique et Technologique Laseris 1, Avenue du Medoc, 33114 Le Barp, France
\end{abstract}

a) Electronic mail: pierre.bejot@u-bourgogne.fr

\begin{abstract}
A simple and compact single-shot autocorrelator is presented and analyzed in detail. The autocorrelator is composed of two elements only: a Fresnel biprism used to create two temporally delayed replicas of the pulse to characterize and a camera in which two-photon absorption takes place. The two-photon absorption signal obtained in the camera can be used to retrieve the pulse duration, the frequency chirp, and the pulse spectrum, provided that a Gaussian temporal shape is assumed. Thanks to its extreme simplicity, the autocorrelator is robust and easy to align. The presented design can theoretically characterize the pulse duration from about 25 fs to 1.5 ps in the two-photon spectral range of the camera (1200-2400 nm). Finally, a proof-of-principle demonstration is also performed at $3.1 \mu \mathrm{m}$ by using an InGaAs camera, whose two-photon spectral range is located further in the infrared (1800-3400 nm).
\end{abstract}

Published under license by AIP Publishing. https://doi.org/10.1063/1.5054357

\section{INTRODUCTION}

The development of optical laser sources able to deliver pulses whose durations are well shorter than the typical time scale of electronic sensors called for the development of all-optical techniques able to characterize the temporal profile of the emitted light. ${ }^{1}$ In this context, strong efforts have been dedicated to develop more and more accurate techniques able to characterize more and more complex laser pulse temporal profiles. Accordingly, numerous ultrashort laser pulse characterization techniques have been developed. Briefly, these techniques can be cast into two main categories, which both have their own advantages and drawbacks. The first one, the simplest in an experimental point of view, is to simply measure a time integrated optical signal which is nonlinear with respect to the electric field to be measured. This signal is produced by two (or sometimes more) replicas of the pulse to be characterized. By varying the relative delay $\tau$ between the replicas, one then can reconstruct a signal $S(\tau)$ called nonlinear autocorrelation. ${ }^{2-7}$ The optical nonlinear effects used for the measurement can be the second or third harmonic generation (SHG/THG), the Kerr effect (polarization gating, self-diffraction), or the two-photon absorption, 3,8, to cite a few. While these techniques are easy to implement, they suffer from two main drawbacks. First, autocorrelators only estimate the pulse duration, provided that a particular temporal pulse shape is assumed. This is because such kinds of techniques only measure the temporal nonlinear autocorrelation of the pulse and not the actual temporal pulse shape directly. As a consequence, since the operation linking a signal and its nonlinear autocorrelation are not bijective, different temporal pulse shapes lead to the same autocorrelation signal. Moreover, autocorrelators are sensitive to the coherent artifact when measuring pulses trains. These two problems have led to the development of more sophisticated methods able to fully reconstruct the pulse temporal shape, such as frequency-resolved optical gating (FROG), ${ }^{10-20}$ spectral phase interferometry for direct electric-field reconstruction (SPIDER), ${ }^{21}$ grating-eliminated no-nonsense observation of ultrafast incident laser light e-fields (GRENOUILLE), ${ }^{22}$ Another Ridiculous Acronym for Interferometric Geometrically Simplified Noniterative E-field Extraction (ARAIGNEE), ${ }^{23}$ and dispersion-scan (D-Scan), ${ }^{24}$ to cite a few. Without going into detail, all these techniques allow to recover both the spectral and temporal phases and amplitudes of the pulse to be measured. In this sense, these techniques turn out to be far 
more complete in terms of temporal characterization than autocorrelators since they do not need any assumption on the temporal pulse profile. However, the price to pay for being able to fully reconstruct the temporal dependence of the electric field is to make the optical design and the practical use of the setup more complex. As a consequence, these techniques are well suited for properly measuring complex and/or very short pulse shapes or pulse trains. On the contrary, optical autocorrelation measurements are often sufficient for a daily and fast characterization (even if incomplete) of "simple" optical pulses delivered, for instance, by low repetition rate chirped-pulsed amplifiers or by secondary sources pumped by amplifiers such as noncollinear optical parametric amplifiers. In particular, noncollinear optical parametric amplifiers operating between 1.2 and $2.4 \mu \mathrm{m}$ pumped by Ti:Sa $800 \mathrm{~nm}$ chirped pulse amplifiers are more and more widespread in laboratories. Generally speaking, there is currently a particular interest for this wavelength region for applications spanning from strong-field physics to optical telecommunications at $2 \mu \mathrm{m}$. In this context, we present here a technique for ultrashort pulse characterization based on single shot interferometric autocorrelation and two photon absorption that we called biprism based optical autocorrelation with retrieval (BOAR). It is composed by only two optical elements: a Fresnel biprism (FB) and a camera, the latter being used as a nonlinear medium and detector at the same time. The broad spectral working range of the presented autocorrelator (1200-2400 nm with a silicon camera and 1800-3400 nm with an InGaAs camera) comes from the broad one-photon response range of the CCD that is placed in the short wavelength range. Moreover, since the nonlinear process is not based on a frequency-conversion process, our autocorrelator is almost insensitive to the input polarization. As every autocorrelators, an assumption on the temporal pulse shape has to be done and our apparatus cannot give a complete temporal characterization of the pulse. Nevertheless, the interferometric nature of the measurement allows, under the linearly chirped Gaussian shape pulse hypothesis, us to retrieve in good approximation the duration, the quadratic phase, and the spectral amplitude of the pulse. The first part of the paper is devoted to the presentation of the optical and mechanical design of the autocorrelator. Then, experimental measurements performed on a commercial noncollinear optical parametric amplifier are presented. In the third part of the paper, the measurements are compared to a theoretical and numerical model mimicking as close as possible the autocorrelator behavior. This model is then used so as to evaluate the working range and accuracy of the BOAR. Finally, it is shown that the presented techniques can be also used for characterizing pulses located even further in the infrared by replacing the silicon camera by an InGaAs one.

\section{BASIC PRINCIPLES OF THE BOAR}

The biprism based optical autocorrelator with retrieval (BOAR) relies on two basic principles: the creation of two identical temporally delayed subpulses from the pulse to be measured and a nonlinear optical effect. These two steps are, respectively, performed with a Fresnel biprism and a camera in which twophoton absorption occurs. The combination of these two elements makes the BOAR extremely compact, calibration-free, and robust in alignment.

\section{A. Geometrical considerations}

Figure 1(a) illustrates the basic principle of the autocorrelator. The creation of the two replica is performed by means of a Fresnel biprism. ${ }^{22}$ For the following discussion, we will consider that the Fresnel biprism has an apex angle $A$, a refractive index $n$, a thickness $e_{0}$, and a height $H$ (it will be supposed hereafter that the laser beam is larger than the biprism, i.e., the biprism is the pupil of the optical system). The propagation of the laser pulse in the biprism leads to the creation of two identical beams crossing with an angle $2 \alpha$ in a plane parallel to the biprism input face, where $\alpha=\operatorname{asin}[n \sin \beta]-\beta$ and $\beta=\pi / 2-A / 2$. The optical path difference in the two beams imposes a temporal delay given in first approximation by $\Delta \tau=\frac{2 x \sin \alpha}{c}$, where $x$ is the distance relative to the propagation axis $z$. The temporal information is then directly transposed in the spatial domain, then allowing single-shot pulse duration measurements, provided that the integration times of the camera is shorter than the repetition period of the laser to be measured. The optimal distance $d_{\mathrm{opt}}$ at which the two beams perfectly spatially overlap is given by $d_{\text {opt }}=\frac{H}{4}\left(\frac{1}{\tan \alpha}-\tan \beta\right)$. At this optimal distance, the temporal window $\Delta \tau_{\max }$ is given by $\Delta \tau_{\max }$ $=\frac{H}{c} \sin \alpha(1-\tan \alpha \tan \beta)$. Note that these formulas are obtained assuming that the laser pulse propagates at phase velocity in both the biprism and air, which leads to a slight underestimation of the pulse duration. This can be corrected by applying a proportionality factor $\kappa=1+\frac{\omega_{0} \cos \alpha}{n \cos \alpha-1} \frac{\partial n}{\partial \omega}$ to the retrieved pulse duration that takes into account the fact that the pulse envelope propagates at the group velocity.
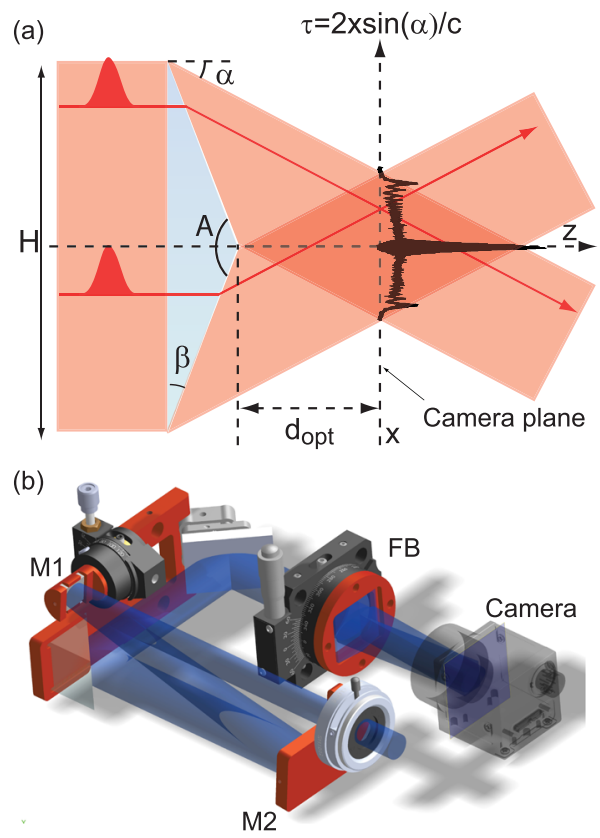

FIG. 1. (a) Principle of the autocorrelator BOAR device. The black line is a typical experimental two-photon absorption signal recorded by using the camera. (b) Experimental setup. The two cylindrical mirrors $M_{1}$ and $M_{2}$ ( $f=-25 \mathrm{~mm}$ and $f=125 \mathrm{~mm}$, respectively) are used for increasing the beam size in the horizontal direction by a factor of 5 , and FB is the Fresnel biprism. 


\section{B. Two-photon absorption}

\section{General formulas}

The optical autocorrelation is performed by two-photon absorption in a camera having a pixel size $d x$ and a number of pixels $N$ along $x$ placed at the distance $d$ from the biprism output. The bandgap $U_{\mathrm{g}}$ of the semiconductor material composing the camera pixels is chosen in the interval $\frac{h c}{\lambda}<U_{\mathrm{g}}<2 \frac{h c}{\lambda}$, where $h$ is the Planck constant, $c$ is the light celerity, and $\lambda$ is the central wavelength to be measured. For instance, a silicon (respectively InGaAs) camera $\left(U_{\mathrm{g}}=1.11 \mathrm{eV}\right)$ can be used for measuring pulse duration in the $1200-2400 \mathrm{~nm}$ (respectively $1800-3400 \mathrm{~nm}$ ) spectral range.

Considering the total complex electric field $\varepsilon(t, \tau)=\varepsilon_{1}(t)$ $+\varepsilon_{2}(t-\tau)$ hitting a pixel of the camera, where $\varepsilon_{1}(t)=A_{1}(t) \mathrm{e}^{i \omega_{0} t}$ [respectively $\varepsilon_{2}(t)=A_{2}(t) \mathrm{e}^{i \omega_{0} t}$ ] is the field at frequency $\omega_{0}=2 \pi c / \lambda$ coming from the upward (respectively downward) face of the biprism, the photocurrent $I_{\text {phot }}$ induced by two-photon absorption on one pixel of the camera is

$$
I_{\text {phot }}(\tau) \propto \int\left|\varepsilon^{2}(t, \tau)\right|^{2} d t .
$$

Assuming a perfect balance between the two replicas' amplitudes $\left(A_{1}=A_{2}=A\right)$, the photocurrent is given by

$$
I_{\text {phot }}(\tau) \propto G_{2}(\tau)+F_{1}(\tau) \cos \left(\omega_{0} \tau\right)+F_{2}(\tau) \cos \left(2 \omega_{0} \tau\right),
$$

where

$$
\begin{aligned}
& G_{2}=2 \int I^{2}(t) d t+4 \int I(t) I(t-\tau) d t, \\
& F_{1}=4 \int[I(t)+I(t-\tau)] \mathcal{R} e\left[A(t) A^{*}(t-\tau)\right] d t, \\
& F_{2}=2 \int \mathcal{R e}\left(A^{2}(t) A^{* 2}(t-\tau)\right) d t,
\end{aligned}
$$

and where $I=|A|^{2}$ is the field intensity. The contributions $G_{2}, F_{1}$, and $F_{2}$ oscillate at very different frequencies $\left(\omega=0, \omega_{0}\right.$, and $2 \omega_{0}$, respectively). Therefore, their relative contributions to the two-photon signal $I_{\text {phot }}$ can be easily distinguished by an appropriate frequency filtering. One has to emphasize that the two-photon absorption signal then can be used to determine the central frequency of the laser to be measured, provided that the optical characteristics of the biprism (Apex angle and refractive index) are well determined. Note that since a part of the autocorrelation evolves with a frequency around $2 \omega_{0}$, the optical system has to be able to resolve the fringes at that frequency, which imposes, in turn, an upper limit on the spatial resolution of the optical system.

\section{Autocorrelation measurement assuming chirped Gaussian pulses}

As every autocorrelator, an assumption about the temporal shape of the pulse has to be done so as to retrieve the pulse duration from the measurement. One of the widely used assumptions is to consider a Gaussian profile, possibly with a quadratic spectral phase. More particularly, if one imposes a quadratic spectral phase $\Phi(\omega)=K\left(\omega-\omega_{0}\right)^{2}$ on a Gaussian electric field with an envelope $A(t)=\mathrm{e}^{\frac{-t^{2}}{\sigma_{t}^{2}}}$ [and its associated spectral amplitude $\widetilde{A}(\omega) \propto \mathrm{e}^{\frac{-\sigma_{t}^{2} w^{2}}{4}}$ ], the electric field complex envelope becomes

$$
A(t)=\mathrm{e}^{\frac{-t^{2}}{\sigma_{t_{c}}^{2}}} \mathrm{e}^{-i a t^{2}},
$$

with $\sigma_{t_{c}}=\frac{\sqrt{\sigma_{t}^{4}+16 K^{2}}}{\sigma_{t}}$ and $a=-4 \frac{K}{\sigma_{t}^{4}+16 K^{2}}$. According to these definitions, $\sigma_{t}$ (respectively $\sigma_{t_{c}}$ ) corresponds to the Fourier-transformed limited (respectively effective) pulse duration, and $a$ is the temporal phase. The defined pulse durations $\sigma_{t}$ (respectively $\sigma_{t_{c}}$ ) are related to full width at half maximum (FWHM) Fourier-transformed limited (respectively effective) pulse durations by

$$
\begin{aligned}
\Delta t_{F W H M} & =\sqrt{2 \log 2} \sigma_{t}, \\
\Delta t_{c, F W H M} & =\sqrt{2 \log 2} \sigma_{t_{c}} .
\end{aligned}
$$

In this case, $G_{2}, F_{1}$, and $F_{2}$ read, respectively,

$$
\begin{aligned}
& G_{2}(\tau)=1+2 \mathrm{e}^{-\frac{\tau^{2}}{\sigma_{t_{c}}^{2}}}, \\
& F_{1}(\tau)=4 \cos \frac{a \tau^{2}}{2} \mathrm{e}^{-\frac{\tau^{2}}{2}\left(\frac{1}{\sigma_{t_{c}}^{2}}+\frac{1}{2 \sigma_{t}^{2}}\right),} \\
& F_{2}(\tau)=\mathrm{e}^{-\frac{\tau^{2}}{\sigma_{t}^{2}}} .
\end{aligned}
$$

A few remarks can then be done regarding the above expressions. First, the low-frequency part of the autocorrelation $\left(G_{2}\right)$ gives access to the chirped pulse duration. However, a chirped pulse and a Fourier-transform limited pulse with the same duration produce the same signal for $G_{2}$. Note that $G_{2}$ embeds an offset induced by the two-photon absorption signal produced by the two replicas taken individually. As discussed below, the presence of this offset can be detrimental for an accurate fit of the pulse duration. Second, the contribution oscillating around $2 \omega_{0}\left(\mathrm{~F}_{2}\right)$ is insensitive to the chirp and gives access to the unchirped pulse duration. More particularly, the amplitude of the Fourier transform of $\mathrm{F}_{2}$ corresponds to the field spectrum amplitude in the case of Gaussian pulses. Finally, the contribution $F_{1}$ oscillating around $\omega_{0}$ is sensitive to both the pulse duration and the chirp. This function, however, is ill-suited in the case of highly chirped pulses. Indeed, this function tends to a distribution that is independent of the chirp applied to the field,

$$
F_{1}(\tau) \underset{K \rightarrow \infty}{\sim} \mathrm{e}^{-\frac{\tau^{2}}{4 \sigma_{t}^{2}}} .
$$

As a consequence, particular care must be taken if one uses this function for retrieving the effective pulse duration, in particular if the spatial profile of the beam is noisy.

To conclude, the pulse duration can be retrieved in two different ways. The first method is to fit $G_{2}$. The drawback of this measurement lies in the fact that this contribution is very sensitive to the quality of the beam profile. Indeed, since this contribution is a low-frequency oscillating function, it is affected by the noise coming from the beam shape imperfections. Moreover, contrary to intensimetric single shot autocorrelators based on a type II second harmonic generation that provide a background free autocorrelation signal, $G_{2}$ includes not only the autocorrelation signal but also the single SHG contribution of each replica beam. This high level of background makes the autocorrelation difficult to fit and especially when this background is noisy and not flat. Finally, the knowledge of $G_{2}$ does not provide any information on the frequency chirp. The second method is to use the combination of $F_{1}$ and $F_{2}$. First, the Fourier-transform limited pulse duration is retrieved 
by fitting $F_{2}$, which also gives the pulse spectrum as described above. Then, the absolute value of the chirp parameter $K$, and consequently, the chirped pulse duration, can be determined by fitting $F_{1}$. As mentioned above, the determination of $K$ is limited to moderate chirps most currently encountered in real experiments. Note that the two methods can be performed simultaneously and independently, improving then the accuracy and reliability of the measurement.

\section{EXPERIMENTAL SETUP AND RESULTS}

The optical design of the autocorrelator is depicted in Fig. 1(b). Before the Fresnel biprism, a cylindrical reflective telescope, composed by two cylindrical mirrors $(f=-25 \mathrm{~mm}$ and $f=125 \mathrm{~mm}$, respectively), increases the beam size by a factor of 5 in the horizontal direction, direction on which the pulse duration measurement is performed. The telescope ensures that the beam size does not limit the range of pulse durations that can be measured by the autocorrelator. Then, $\mathrm{a} A=160^{\circ} 1.7 \mathrm{~mm}$ thick fused silica biprism is mounted on a manual rotation stage. Using this biprism and after correction coming from the difference between phase and group velocities, the total delay range $\Delta \tau_{\max }$ is then $\Delta \tau_{\max } \simeq 5.5 \mathrm{ps}$ and the optimal distance is $d_{\mathrm{opt}}=6.3 \mathrm{~cm}$. The camera used in the autocorrelator is a 12 bit silicon CMOS camera. The minimal integration time is $35 \mu \mathrm{s}$, which makes the autocorrelator single-shot for lasers with a repetition rate lower than $56 \mathrm{kHz}$. The camera can be externally triggered with a $14 \mathrm{~Hz}$ maximal frame rate. The pixel size of the camera is $\mathrm{dx}=1.67 \mu \mathrm{m}$, which allows us to resolve the optical fringes in the whole spectral range of interest. In the horizontal direction, the camera has 3840 pixels, which gives a $6.4 \mathrm{~mm}$ sensor size. Accordingly, the total delay range accessible with the present camera is only $3.5 \mathrm{ps}$, while the upper limit imposed by the biprism is 5.5 ps. As a consequence, since the camera field of view actually limits the delay range, the biprism was placed at a distance $d=4.7 \mathrm{~cm}$, i.e., before $d_{\text {opt. }}$. The rotation of the biprism is adjusted by aligning the interference fringes along the vertical dimension of the camera. The signal is transferred by universal serial bus (USB) 3.0 to a computer for further processing. While the refractive index of the material composing the biprism (in the present case, fused silica) is well known, the calibration of the apex angle has been accurately performed prior to any autocorrelation measurements by illuminating the optical system with a $\mathrm{HeNe}$ laser and by measuring the interference fringes spacing on the camera. The calibration procedure finally led to $A=160.220^{\circ} \pm 0.005^{\circ}$. The laser characterized during the experiment is a commercial noncollinear optical parametric amplifier pumped by a 100 fs $800 \mathrm{~nm}$ chirped pulse amplified laser and delivers femtosecond laser pulses in the 1200-2400 nm wavelength region. Figure 2(a) shows a typical signal recorded with the autocorrelator for a laser wavelength set at $\lambda_{0}=2020 \mathrm{~nm}$. A fast Fourier transform along the horizontal dimension allows us to easily separate the three oscillating functions $G_{2}, F_{1}$, and $F_{2}$ that are shown in Figs. 2(b)-2(d). At the same time, knowing that $F_{1}$ and $F_{2}$ oscillate around $\omega_{0}$ and $2 \omega_{0}$, respectively, the central wavelength of the laser is estimated to be $\lambda_{\mathrm{e}}=2022 \mathrm{~nm}$, in very good agreement with the expectations. The retrieval procedure applied to $G_{2}$ gives a pulse duration (FWHM) of $\Delta t \simeq 76 \mathrm{fs}$. Using $F_{1}$ and $F_{2}$, one obtains $\Delta t \simeq 77 \mathrm{fs}$ (in very good agreement with the result obtained with $G_{2}$ ), a Fourier-transform limited pulse duration of

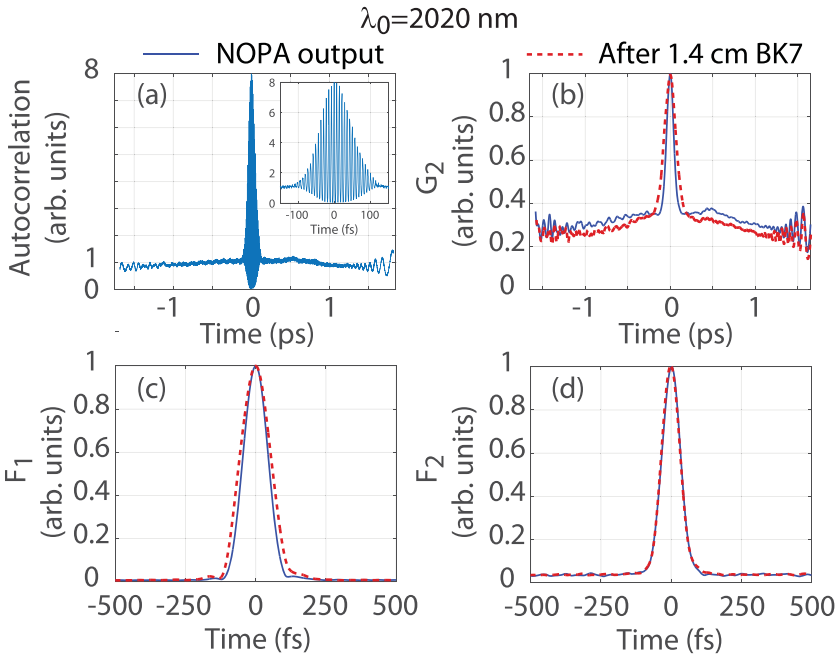

FIG. 2. Autocorrelation obtained for a pulse at $2020 \mathrm{~nm}$ (a) and the associated oscillating function $G_{2}(b), F_{1}(c)$, and $F_{2}(d)$. The measurement of the pulse duration performed at the output of the NOPA is shown in blue line, while those done after propagation in a $1.4 \mathrm{~cm} \mathrm{BK7}$ plate is depicted in dashed red line.

$\Delta t_{\mathrm{FTL}} \simeq 57 \mathrm{fs}$ and a group delay dispersion (GDD) of $\pm 1050 \mathrm{fs}^{2}$. Then, the same procedure has been performed after having inserted a $1.4 \mathrm{~cm}$ thick BK7 plate in the laser path [Figs. 2(b)-2(d)]. As expected, the contribution oscillating around $2 \omega_{0}$ remains unchanged, while both $G_{2}$ and $F_{1}$ get broader than in the case without the plate. The fit of $G_{2}$ leads to $\Delta t \simeq 137 \mathrm{fs}$, while those of $F_{1}$ and $F_{2}$ lead to $\Delta t \simeq 145 \mathrm{fs}$, a Fourier-transform limited pulse duration of $\Delta t_{\mathrm{FTL}} \simeq 56.7 \mathrm{fs}$ and a group delay dispersion (GDD) of $\pm 2720 \mathrm{fs}^{2}$. Accordingly, one can estimate that the absolute value of the GDD introduced by the BK7 plate is about $1670 \mathrm{fs}^{2}$. This results is in good agreement with the theoretical expectation $\left(-1450 \mathrm{fs}^{2}\right)$ calculated from the Sellmeier formula, in particular if one remembers that assumptions have been made on the pulse temporal and spectral profiles.

As discussed in Sec. II B 2, $F_{2}$ can be used for estimating the laser spectrum. In order to confirm this, the spectrum retrieved with the autocorrelator has been compared with those actually measured with a commercial spectrometer in the case of a laser pulse centered around $\lambda_{0}=1820 \mathrm{~nm}$. As shown in Fig. 3, the spectrum estimated by the autocorrelator agrees well with the spectrum measured with the commercial spectrometer.

Usually, autocorrelators are sensitive to the input polarization. This is because almost all autocorrelators are based on harmonic generation, which is a strongly polarization-dependent process because of the phase-matching issue. Accordingly, in such apparatus, the input pulse has to be linearly polarized in a particular direction. Since the presented autocorrelator is based on a different process (two-photon absorption), one can wonder which polarization has to be used during the measurement. The two-photon absorption process is a priori a polarization dependent process due to the tensor nature of the two-photon absorption cross section. In order to check the polarization dependence, a Berek compensator has been inserted before the autocorrelator. This compensator allows us to impose any 


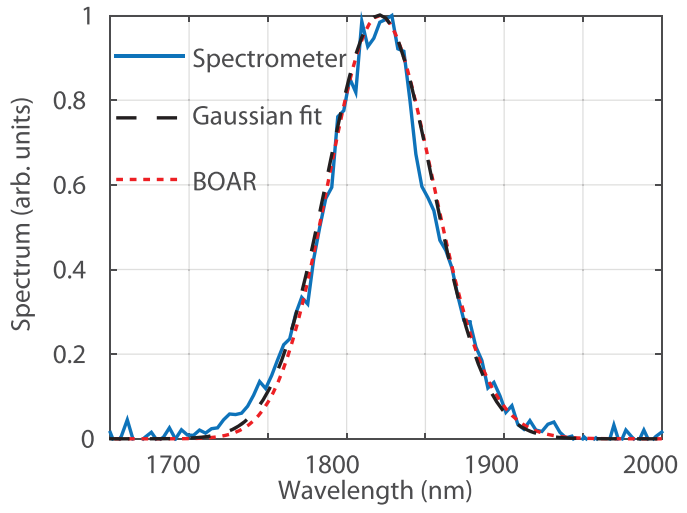

FIG. 3. Laser spectrum measured with a commercial spectrometer (blue solid line) and evaluated with the BOAR (red dotted-dashed line). The black dashed line is a Gaussian fit of the spectrum measured with the spectrometer. The central wavelength of the NOPA is set to $1820 \mathrm{~nm}$.
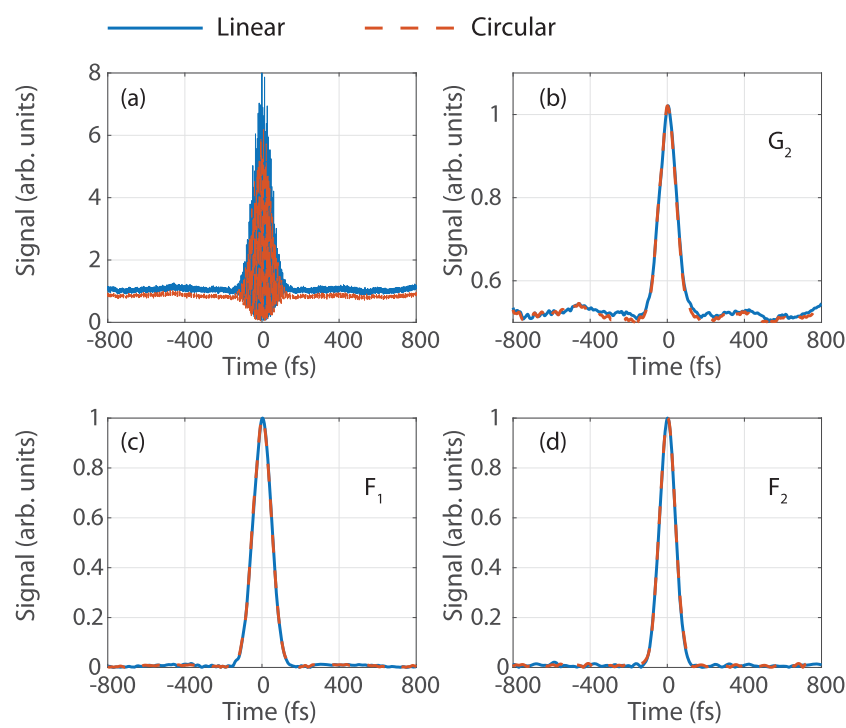

FIG. 4. Comparison between experimental autocorrelations obtained for a linearly (dashed red) and circularly (solid blue) polarized pulse (a) and the associated oscillating functions $G_{2}$ (b), $F_{1}$ (c), and $F_{2}$ (d) in the case of a $1400 \mathrm{~nm}$ laser pulse.

polarization to the pulse to be measured. A comparison between the linear and circular polarizations is depicted in Fig. 4. Apart from a slightly less intense signal obtained in the circular case, the two measured autocorrelations are almost identical and the fitting procedure gives the same results in both cases. Note that the autocorrelator has also been tested in the cases of elliptical polarizations and gives the same result.

\section{FULL-SPATIOTEMPORAL DYNAMICS}

Since the temporal characteristics of the pulse are transposed in the spatial domain, the spatial beam shape can affect the autocorrelation measurement. It is then of prime importance to characterize the spatiotemporal shape of the laser pulse in particular at the plane at which the camera is placed. To this end, full spatiotemporal propagation simulations have been performed so as, first, to validate the experimental protocol and, second, in order to quantify potential limitations of the autocorrelator. In order to mimic as close as possible, the experimental configuration, a $H=2 \mathrm{~cm}$ fused silica biprism with $160^{\circ}$ apex angle, has been considered. Accordingly, the expected delay range accessible with this biprism is about $\Delta \tau_{\max }=5$ ps and the optimal distance is $d_{\text {opt }} \simeq 6.3 \mathrm{~cm}$. The central wavelength of the laser has been set to $\lambda_{0}=2 \mu \mathrm{m}$. The spectrum $\widetilde{E}(x, \omega)$ of the electric field $E(x, t)$, chosen to be as close as possible to the experimental conditions presented here, reads in good approximation after the biprism,

$$
\widetilde{E}(x, \omega, d=0)=E_{0} \mathrm{e}^{-\frac{x^{6}}{\sigma_{p}^{6}}} \mathrm{e}^{-\frac{x^{2}}{\sigma_{x}^{2}}} \mathrm{e}^{-\frac{\sigma_{t}^{2}\left(\omega-\omega_{0}\right)^{2}}{4}} \mathrm{e}^{i K\left(\omega-\omega_{0}\right)^{2}} \mathrm{e}^{i \Phi(x, \omega)} .
$$

The super-Gaussian function $\left(\sigma_{p}=0.98 \mathrm{~cm}\right)$ additionally superimposed to the spatial profile mimics the geometrical truncation induced by the biprism, the latter being smaller than the beam size $\left(\sigma_{x}=3.4 \mathrm{~cm}\right)$ after its magnification by the cylindrical telescope. Finally, $\Phi(x, \omega)$ is the frequency-dependent phase introduced by propagation through the biprism. The latter reads

$$
\Phi(x, \omega)=n(\omega) \omega e(x) / c+\omega l(x) / c,
$$

where $e(x)$ [respectively $l(x)$ ] is the thickness of the fused silica (respectively air) crossed by the ray arriving at a given height $x$ at the output of the biprism,

$$
\begin{aligned}
& e(x)=\left(\frac{H}{2}-\frac{|x|}{1-\tan [\alpha(\omega)] \tan \beta}\right) \tan \beta, \\
& l(x)=|x| \frac{\sin \beta}{\cos [\alpha(\omega)+\beta]} .
\end{aligned}
$$

The introduced phase then takes into account the fact that the central part of the laser pulse experiences a stronger dispersion than the outer region because of a longer propagation path through the biprism. It also takes into account, through the frequency dependence of $\alpha$, that the frequencies within the pulse spectrum are not deviated exactly with the same angle because of the refractive index dispersion, which leads to spatiotemporal couplings during the propagation after the biprism. These two parameters, together with the finite extension of the two-photon absorption band, are those limiting the measurement of very short laser pulses as it will be described below. After a propagation distance $d$ in the air, the electric field becomes in the Fourier space,

$$
\widetilde{E}\left(k_{x}, \omega, d\right)=\widetilde{E}\left(k_{x}, \omega, 0\right) \mathrm{e}^{i \sqrt{k_{\text {air }}^{2}(\omega)-k_{x}^{2}} d},
$$

where $k_{\text {air }}(\omega)=n_{\text {air }}(\omega) \omega / c, k_{x}$ is the conjugate variable of $x$ in the reciprocal space, and $n_{\text {air }}$ is the frequency-dependent refractive index of the air. The spatiotemporal distribution of the electric field $E(x, t, d)$ is then easily retrieved by an inverse Fouriertransform in both space and time. Figure 5 shows the spatiotemporal distribution of the pulse intensity at $d=d_{\text {opt }}$. The two parts of the beam coming from the two faces of the biprism intersect and interfere at the center because they temporally overlap. Note that the part of the energy located at positive times comes from the 


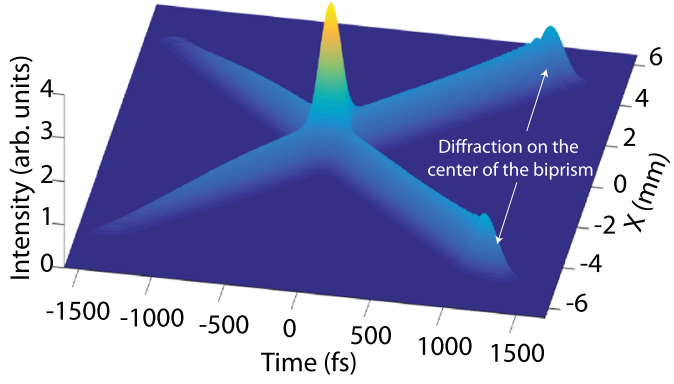

FIG. 5. Spatiotemporal distribution of the pulse intensity at $d=d_{\mathrm{opt}}$.

central edge of the biprism. Because of the discontinuity, it experiences diffraction so that oscillations can be noticed in the spatial profile. The photocurrent induced by two-photon absorption is then calculated by using Eq. (1) as exemplified in Fig. 6(a) for $d=d_{\mathrm{opt}}=6.3 \mathrm{~cm}$ in the case of a $\lambda_{0}=2 \mu \mathrm{m} 100$ fs Fourier transform limited laser pulse. Then, the three oscillating functions $F_{2}, F_{1}$, and $G_{2}$ are isolated by a spectral filtering [Fig. 6(c)] and fitted using Eq. (7). As explained earlier, working at $d=d_{\text {opt }}$ provides the largest temporal window. However, because of the Gaussian spatial profile, the offset is not uniform at this distance, which can be detrimental to correctly fit $G_{2}$ using Eq. (7). For solving this issue and since the camera size actually limits the duration range that can be measured, a slightly shorter distance $(d=4.7 \mathrm{~cm})$ was chosen experimentally so as to obtained a flat offset over the full temporal window. The numerical two-photon absorption signal obtained in this case and the associated oscillating functions are shown in Figs. 6(b) and 6(d), respectively. Finally, the output of the propagation code has been compared to the experiments (see Fig. 7). The pulse parameters chosen in the simulation are those retrieved by the fit procedure during the experiment $\left(\Delta t_{\mathrm{FTL}}=57 \mathrm{fs}\right.$ and $\left.\Delta t=77 \mathrm{fs}\right)$. As shown, the simulation is in excellent agreement with the experiment, which then validates the propagation code.

\section{A. Limitations of the autocorrelator}

\section{Theoretical pulse duration working range}

Several distinct factors limit the pulse duration range that can be measured by means of the presented autocorrelator. The minimal Fourier-transfom-limited (FTL) pulse duration that can be measured is limited by the group velocity dispersion occurring during the propagation through the biprism (even if this spectral phase can be more or less compensated by a pulse compressor). In the case of a Fourier transform limited pulse, the propagation within the biprism will make the pulse longer. More particularly, it can be shown that the quadratic phase introduced by the biprism $\Phi_{p}$ is in good approximation,

$$
\Phi_{p}=\frac{k^{(2)}\left(\omega_{0}\right)}{2}\left(\omega-\omega_{0}\right)^{2} e_{\mathrm{eff}}
$$

where $k^{(2)}\left(\omega_{0}\right)=\partial^{2} k / \partial \omega^{2}\left(\omega_{0}\right)$ is the group velocity dispersion of the biprism material, $e_{\mathrm{eff}}=e_{0}\left(1-\frac{d}{2 d_{\mathrm{opt}}}\right)$ is the effective thickness of the biprism, and $e_{0}=\frac{H}{2} \tan \beta$ is the thickness of the biprism at the center. The propagation code described above was used to quantify the minimal and maximal pulse durations that can be measured with our autocorrelator. For a given and known initial set of parameters (FTL pulse duration and frequency chirp), the autocorrelation signal was numerically evaluated, giving in turn the three contributions $G_{2}, F_{1}$, and $F_{2}$ after an appropriate spectral filtering. Then, these extracted contributions were fitted as it is done in experiments. The resulting fits then give access to the evaluated FTL pulse duration and frequency chirp, which can be compared to the initial set of parameters. The difference between the initial conditions
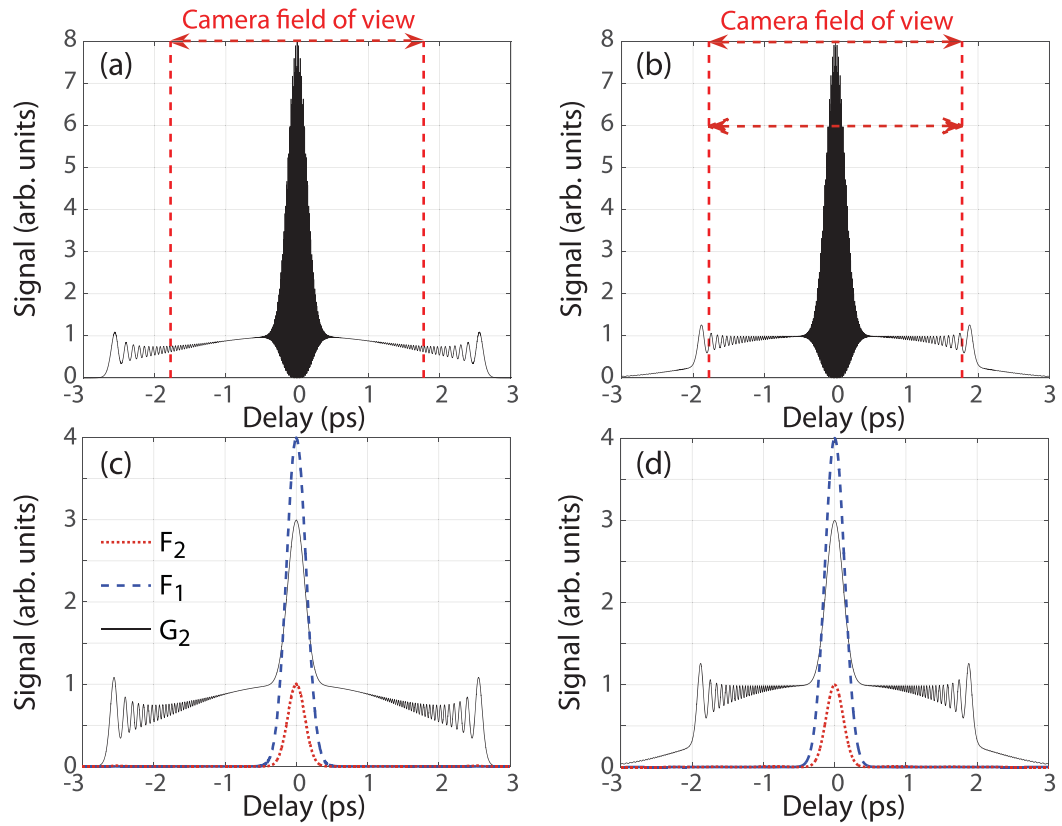

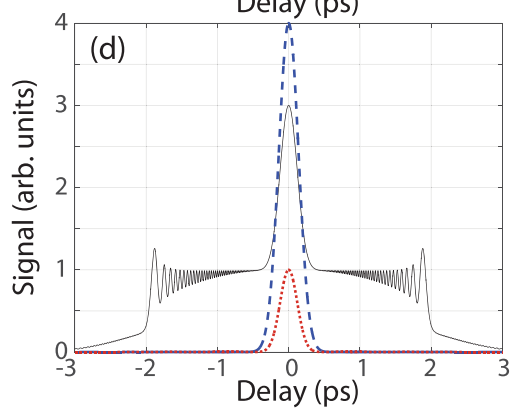

FIG. 6. Typical theoretical autocorrelation signal obtained by two-photon absorption with a $160^{\circ}$ fused silica biprism (a) placed at the optimal distance $\left(d_{\text {opt }}=6.3 \mathrm{~cm}\right)$ and $(\mathrm{b})$ where the biprism is placed experimentally $(d=4.7 \mathrm{~cm})$ for a $\lambda$ $=2 \mu \mathrm{m}, 100 \mathrm{fs}$ pulse. The associated oscillating functions obtained after a spectral filtering are shown in (c) and (d), respectively. 

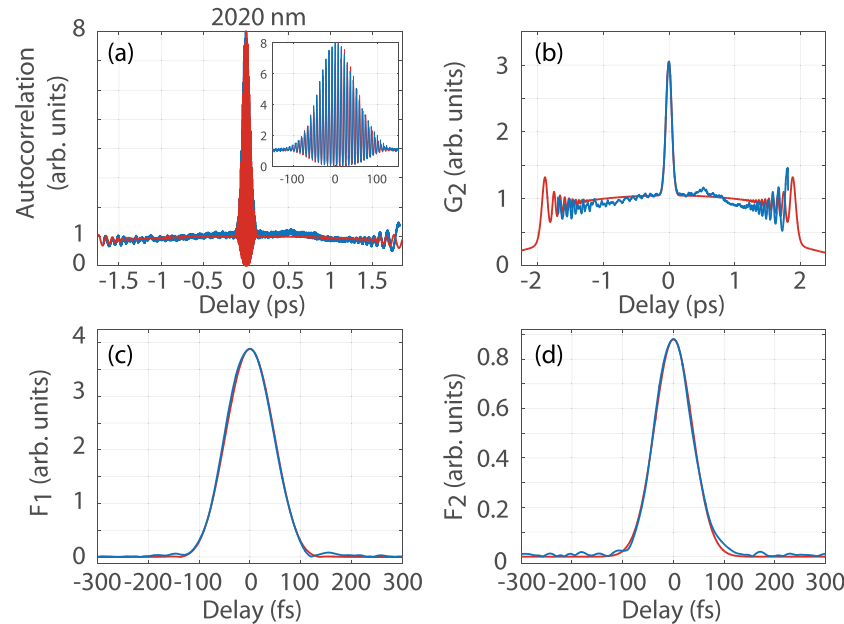

FIG. 7. (a) Comparison between the autocorrelations obtained by our propagation code (red line) and the experiment (blue line). The Fourier-transform limited (respectively chirped) pulse duration of the pulse is 57 (respectively 77) fs. The central wavelength is $2020 \mathrm{~nm}$. Numerical (red line) and experimental (blue line) contributions $G_{2}(b), F_{1}(c)$, and $F_{2}(d)$ retrieved from the autocorrelations shown in (a).

and the fit of the parameters then gives the error introduced by the measurement apparatus itself. Figure 8(a) shows the relative error made by the pulse duration measurement as a function of the input pulse duration (full width at half maximum) in the conditions used during our experiment. The measurement of $F_{2}$ (FTL pulse duration) is almost not affected by the propagation through the biprism. This is because $F_{2}$ is insensitive to the chirp and consequently to the
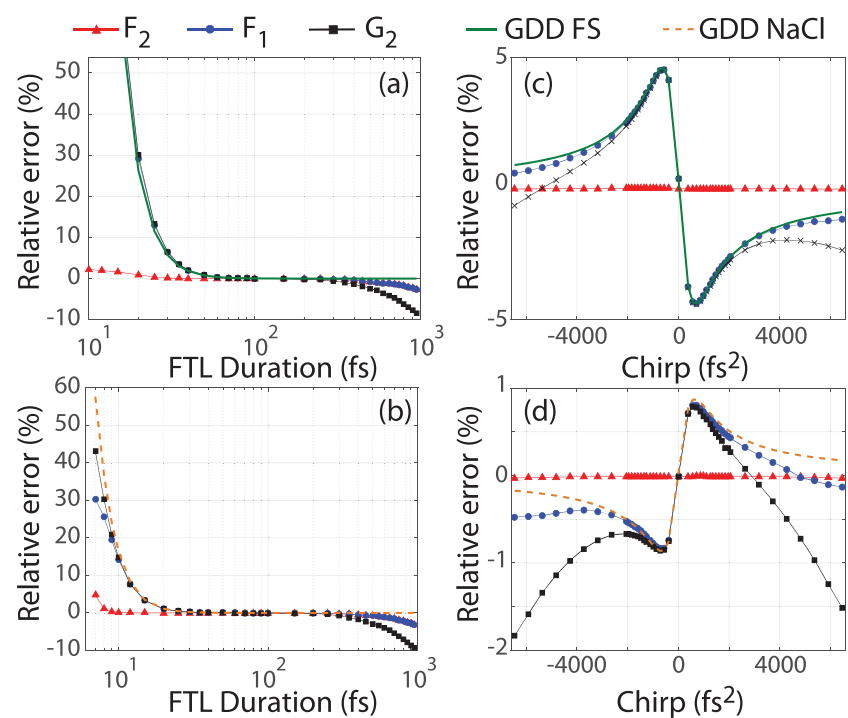

FIG. 8. Relative error made by the fit as a function of the FTL pulse duration in the case of a fused silica (a) and $\mathrm{NaCl}(\mathrm{b})$ prism. Relative error made by the fit as a function of the initial chirp in the case of a fused silica (c) and $\mathrm{NaCl}$ (d) prism for a $60 \mathrm{fs}$ laser pulse. The central wavelength of the pulse is $2 \mu \mathrm{m}$. group velocity dispersion induced in the biprism. In fact, the only thing that introduces an error is the frequency-dependence of the refraction angle at the biprism output leading to an angular chirp. However, this effect remains marginal for pulses longer than $15 \mathrm{fs}$ if a fused silica biprism is used. On the contrary, the retrieved chirped pulse duration (using either $G_{2}$ or $F_{1}$ ) is longer than expected, in particular, for very short pulses and well fits the duration expected if one considers the dispersion induced by the propagation within the biprism. This means that the main effect introducing an error in the pulse measurement comes from the dispersion introduced by the propagation within the biprism and can be easily compensated during the fit algorithm. If the tolerance is set to $10 \%$, the minimal pulse duration that can be measured in this condition (i.e., with a fused silica biprism) is about 25 fs. More particularly, setting a $10 \%$ tolerance in the pulse duration measurement and considering the biprism-induced group velocity dispersion only, one can show that the minimal pulse duration (FWHM) measurable with the present setup is given by

$$
\Delta_{t_{\text {min }}}\left(\omega_{0}\right)=2 \sqrt{\log (2) k^{(2)}\left(\omega_{0}\right) e_{\mathrm{eff}}}\left(1.1^{2}-1\right)^{-1 / 4} .
$$

Since the group-velocity dispersion of the biprism is not constant over the full spectral window, the minimal pulse duration depends on the pulse central wavelength to be measured (Fig. 9). Note that, this limit can be pushed below by the use of a $\mathrm{NaCl}$ biprism instead of a fused silica biprism because the former has a smaller group velocity dispersion beyond $1.6 \mu \mathrm{m}$. Figure 8(b) shows the error made when such a biprism is used (the apex angle of the latter has been chosen so as to keep the same deviation angle, i.e., $A \simeq 163,3^{\circ}$ ). In this case, the minimal theoretical pulse duration measurable is about $11 \mathrm{fs}$. As far as the maximal measurable FTL pulse duration is concerned, it is limited by the finite spatial expansion of the laser beam and does not depend on the biprism material as it can be noticed by comparing Figs. 8(a) and 8(b) in the long pulse region. In the chosen configuration, the maximal pulse duration that can be measured

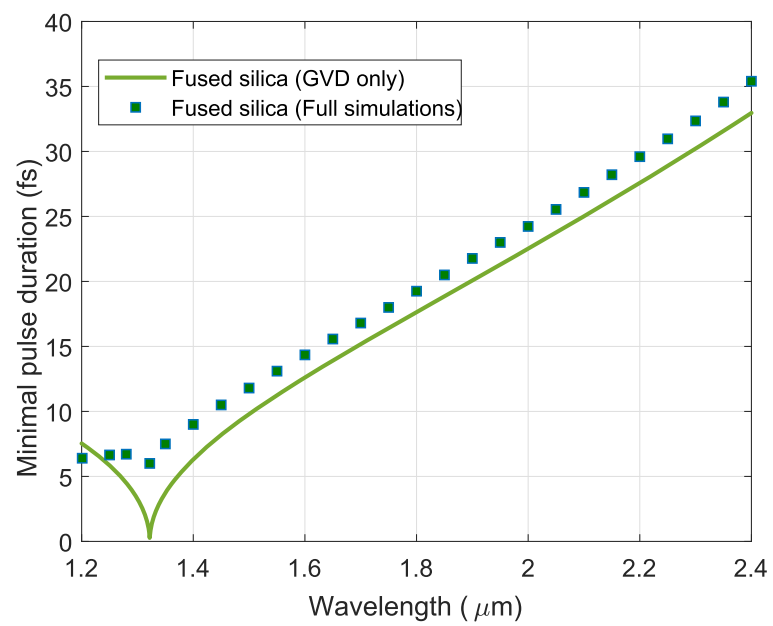

FIG. 9. Theoretical minimal FTL pulse duration measurable (less than $10 \%$ error in the retrieval) with the present autocorrelator as a function of the pulse central wavelength if one only considers the group velocity dispersion of the biprism (solid green line) and according to the full simulations (green squares). 
is about 1 ps (FWHM) when using $G_{2}$ and slightly more ( $\left.\simeq 1.5 \mathrm{ps}\right)$ when using the combination of $F_{1}$ and $F_{2}$. This difference is due to the fact that the quality of the fit of $G_{2}$ does depend on the offset flatness, while those of $F_{1}$ and $F_{2}$ do not. In the case of a chirped laser pulse to be measured, the group delay dispersion induced by the propagation through the biprism impacts the retrieval of the real pulse duration, while the FTL pulse duration retrieval by means of $F_{2}$ remains accurate. Indeed, the quadratic phase after propagation within the biprism $\Phi_{p}$ is

$$
\Phi_{p}=\left(K+\frac{k^{(2)}\left(\omega_{0}\right)}{2} e_{\mathrm{eff}}\right)\left(\omega-\omega_{0}\right)^{2},
$$

where $K$ is the quadratic phase parameter of the pulse to be measured. Depending on the relative sign of $K$ and the group delay dispersion introduced by the biprism, the chirped pulse duration effectively measured by the autocorrelator will be either longer or shorter than the pulse duration of the input pulse. Figures 8(c) and 8 (d) show the relative error of the estimation as a function of $K$ for a $60 \mathrm{fs}$ (FWHM) $2 \mu \mathrm{m}$ laser pulse when using a fused silica or a $\mathrm{NaCl}$ biprism. For small $K$, the error is well fitted by the error introduced by the biprism dispersion. In the highly chirped case, however, the limiting factor is not the group velocity dispersion of the biprism but the finite spatial extension of the laser beam. As it is the case for FTL laser pulse, the use of $G_{2}$ for estimating the laser pulse duration leads to the underestimation of the pulse duration in this case. Note also that the use of a $\mathrm{NaCl}$ biprism leads to a smaller error on the pulse estimation also in this case. It then seems that $\mathrm{NaCl}$ should be preferred for the design of the autocorrelator. However, apart from the case of very short pulses ( $<20 \mathrm{fs})$, fused silica biprism remains a good choice, in particular if one takes into account the fact that $\mathrm{NaCl}$ biprisms are much more costlier than the silica one.

While the calculations presented above evaluate how the present autocorrelator modifies the pulse to be measured because of the biprism dispersion, it is common in real experiments to externally compress the pulse so as to measure the minimal pulse duration at the level of the autocorrelator itself. It is then interesting to evaluate the minimal pulse duration measurable if one tries to compensate the quadratic spectral phase induced by the propagation within the biprism. In this case, the remaining limitation will come from the higher-order dispersion terms induced by the propagation within the biprism and the angular chirp induced by the spectral dependence of the deviation angle $\alpha$ of the biprism (coming from the spectral dispersion of the refractive index). As shown in Fig. 10(a), both the third-order dispersion and the angular chirp are more important at longer wavelengths for the fused silica biprism. As a consequence, as shown in Fig. 10(b), while the theoretical minimal pulse duration measurable by the autocorrelator after compression is slightly more than 6 fs at shorter wavelengths, it increases up to about $16 \mathrm{fs}$ at $2.4 \mu \mathrm{m}$. However, we do not overlook the fact that for such short pulse duration, the assumption of having a Gaussian temporal and spectral profile in real experiment becomes relatively questionable. Comparing the results with those presented in Fig. 9, it appears that higher-order dispersion and angular chirp do not play a role below $1.3 \mu \mathrm{m}$. Finally, one has to emphasize that all these calculations do not take into account the finite spectral extension of the two-photon absorption band.
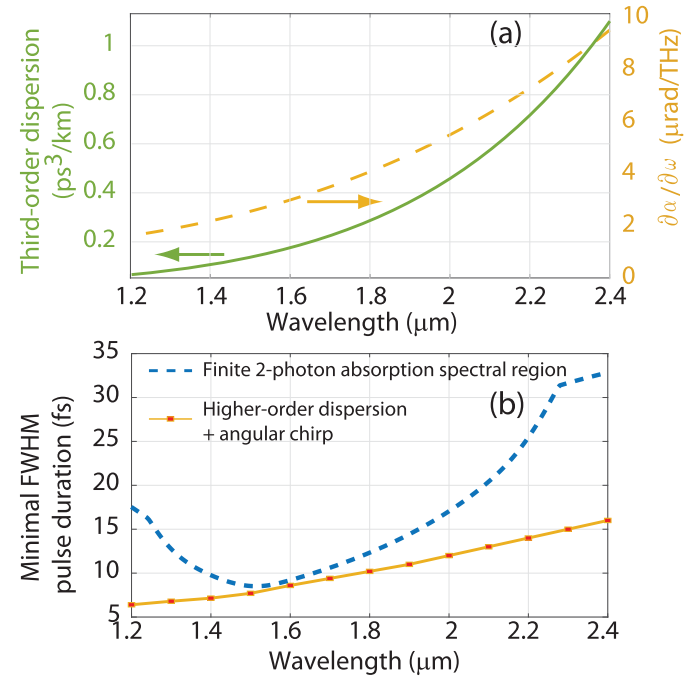

FIG. 10. (a) Third-order spectral dispersion of fused silica (green solid line) and frequency derivative of the deviation angle (orange dashed line). (b) Minimal pulse duration measurable with the autocorrelator if one tries to compensate for the quadratic spectral phase induced by the propagation within the biprism (orange line with red squares) and minimal pulse duration supported by the finite spectral bandwidth of the silicon (dashed blue line).

In particular, close to the upper and lower limit of the band, the minimal pulse duration measurable with our autocorrelator will be limited by the fact that a part of the pulse spectrum (which is broader and broader as the pulse duration decreases) goes beyond the spectral region of two-photon absorption. Figure 10 (b) displays the minimal pulse duration supported by the two-photon absorption region of the silicon. In the first approximation, it has been considered that the two-photon absorption probability is flat over the region 1.15-2.45 $\mu \mathrm{m}$ and then decreases sharply out of this spectral range. Over the full spectral region, it appears that the limiting factor of the full apparatus is indeed the finite spectral bandwidth of the two-photon absorption, even in the center of the spectral band.

\section{Impact of the spatial quality of the beam}

Another experimental parameter affecting the accuracy of a single-shot autocorrelator is the spatial beam quality. Indeed, since the temporal information is transposed in the spatial domain, the presence of noise in the spatial domain in turn degrades the quality of the pulse duration retrieval. In order to estimate the error made when dealing with a noisy laser beam, simulations have been performed with spatial transverse profiles on which different noise amplitudes have been superimposed. The noise used in the simulation has not been chosen as a completely random function but behaves as $1 / k_{x}$ (where $k_{x}$ is the spatial frequency), i.e., a low frequency spatial noise. This choice reproduces somehow the behavior of the noise effectively affecting amplified laser pulses as it has also been verified with our own laser system. Figure 11 shows the relative error made by the fitting procedure for different noise amplitudes (keeping constant the noise spatial shape) as a function of the chirp amplitude for a $60 \mathrm{fs} 2 \mu \mathrm{m}$ laser pulse. The spatial profile of the 

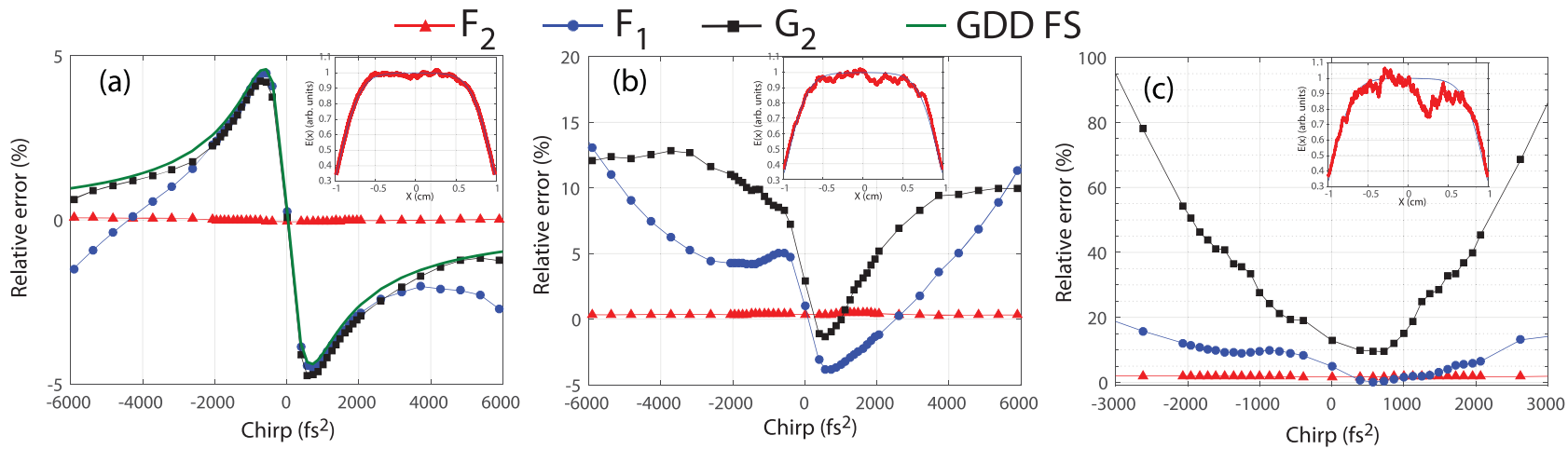

FIG. 11. Relative error made by the fit as a function of the initial chirp in the presence of a low (a), intermediate (b), and strong (c) noise in the spatial profile for a fused silica $160^{\circ}$ biprism. The central wavelength of the pulse is $2 \mu \mathrm{m}$, and the FTL pulse duration is $60 \mathrm{fs}$. The associated spatial electric field profile is shown in the subpanels.

electric field used in the three cases is depicted in the insets. As expected, the presence of noise is detrimental for the pulse duration retrieval. At low noise amplitude [Fig. 11(a)], the error remains limited by the dispersion and finite spatial extend of the laser pulse. This is not the case anymore for intermediate and strong noise amplitudes. It can be noticed that $F_{2}$ is almost unaffected by the presence of noise unlike $F_{1}$ and $G_{2}$. There are two different reasons explaining the different impact of the noise on the fit accuracy. First, one has to remember that $G_{2}, F_{1}$, and $F_{2}$ evolve around $\omega=0, \omega=\omega_{0}$, and $\omega=2 \omega_{0}$, respectively. This difference in the temporal domain is transposed in the spatial domain by the use of the biprism. Since the noise mostly affects the low spatial frequency, it impacts more $G_{2}$ than $F_{1}$ and leaves $F_{2}$ almost unaffected. The second reason explaining why $F_{2}$ is far less impacted than the two other functions is that $G_{2}$ and $F_{1}$ extend on a longer transverse dimension as the chirp parameter increases. As a consequence, they are more sensitive to the low-frequency spatial noise. Finally, as discussed above, the fit accuracy of $F_{1}$ decreases for long chirped laser pulse because this function tends to a chirp unsensitive function as the chirp increases. This is particularly true in the presence of noise on the spatial profile. Note, however, that the impact of the noise can be decreased experimentally by a binning of the pixels over the vertical dimension, which is not used for pulse duration retrieval. This averaging can be directly performed in the hardware of the camera and/or a software-performed averaging over the vertical dimension of the two-photon absorption signal. As shown in Fig. 11, the presence of noise in the spatial profile mostly impacts the accuracy of the fit of $G_{2}$ mainly because the fitting procedure of the latter is strongly dependent on the offset flatness.

\section{EXTENSION OF THE PRINCIPLE AT LONGER WAVELENGTHS}

As explained above, using a silicon camera allows us to measure pulse durations for pulses located between $1200 \mathrm{~nm}$ and $2400 \mathrm{~nm}$, which is the wavelength range of two-photon absorption of the silicon. Measuring the pulse duration of pulses located at higher wavelengths needs to adapt the materials composing the detector. A proof-of-principle experiment was performed by using an InGaAs camera. The camera sensor is composed by $640 \times 51215 \mu \mathrm{m}$ pixels. The $\mathrm{NaCl}$ Fresnel biprism used in this experiment has $175^{\circ}$ apex angle. Figure 12(a) shows the two-photon absorption signal recorded by using the camera in the case of a $3.1 \mu \mathrm{m}$ femtosecond laser. As shown in Fig. 12(b) that displays the Fourier-transform of the signal, the three contributions $G_{2}, F_{1}$, and $F_{2}$ clearly appear, which confirms the two-photon nature of the process. By fitting the oscillating functions $F_{1}$ and $F_{2}$ [see Fig. 12(c)], the pulse is found to be almost Fourier-transform limited with a duration of approximately $95 \mathrm{fs}$.
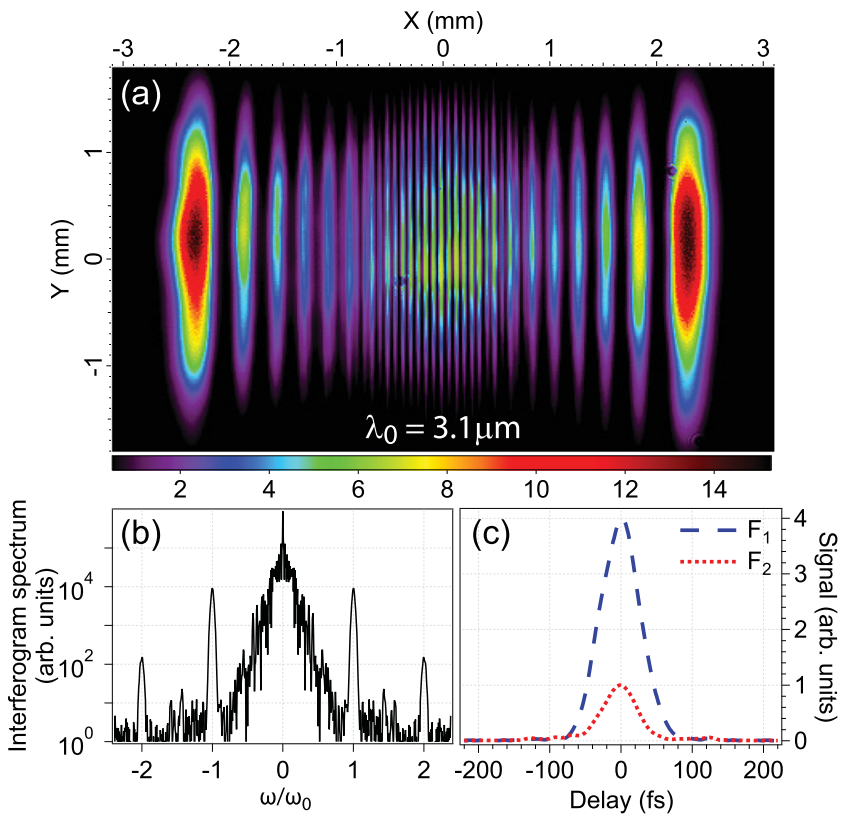

FIG. 12. Pulse measurement performed at $\lambda_{0}=3.1 \mu \mathrm{m}$ with a InGaAs camera. (a) Two-photon absorption signal captured with the InGaAs camera. (b) FourierTransform of the two-photon signal showing the three different contributions oscillating at $\omega=0, \omega_{0}$, and $2 \omega_{0}$, respectively. (c) Temporal distribution of $F_{1}$ and $F_{2}$. The retrieved pulse duration is about $95 \mathrm{fs}$. 


\section{CONCLUSION}

As a conclusion, a simple and compact single-shot autocorrelator based on two-photon absorption in a camera and working in the 1.2-2.4 $\mu \mathrm{m}$ spectral region has been presented and analyzed in detail. The use of a Fresnel biprism for generating the two replicas makes the autocorrelator extremely robust in alignment. Moreover, it has been shown that the autocorrelator is insensitive to the input pulse polarization contrary to almost all existing autocorrelators. The interferometric nature of the autocorrelator allows us also to extract more information than with a conventional intensimetric autocorrelator. In particular, it has been shown that the pulse spectrum, the frequency chirp, and the pulse duration can be retrieved if one assumes a particular temporal shape. Using a propagation code, the limitations of the autocorrelator have been analyzed. In particular, using a fused silica biprism, it has been shown that the pulse duration range accessible with the presented configuration theoretically extends from 25 fs to about 1 ps. Note that the lower limit can even be lowered by using a $\mathrm{NaCl}$ biprism instead of a fused silica one or by using a bimirror instead of a Fresnel biprism (i.e., by using an all-reflective geometry). Nevertheless, we do not overlook that real experiments have to be performed so as to validate the theoretical expectations, in particular in the case of very short pulses. Finally, the extension to higher wavelengths (up to $3.4 \mu \mathrm{m}$ ) has been demonstrated by substituting the silicon camera by an InGaAs one.

\section{ACKNOWLEDGMENTS}

This work was supported by the Conseil Régional de Bourgogne (PARI program), the CNRS, the FEDER-FSE Bourgogne 2014/2020, the Labex ACTION program (Contract No. ANR-11-LABX-0001$01)$, the SATT Grand-Est, and the Carnot-ARTS. We thank the CRM-ICB (Brice Gourier, Julien Lopez, and Jean-Marc Muller) for the mechanical realization of the autocorrelator and the CRI-CCUB for CPU loan on the multiprocessor server.

\section{REFERENCES}

'I. A. Walmsley and C. Dorrer, "Characterization of ultrashort electromagnetic pulses,” Adv. Opt. Photonics 1, 308-437 (2009).

${ }^{2}$ J.-C. M. Diels, J. J. Fontaine, I. C. McMichael, and F. Simoni, "Control and measurement of ultrashort pulse shapes (in amplitude and phase) with femtosecond accuracy," Appl. Opt. 24(9), 1270 (1985).

${ }^{3}$ G. Szabo, Z. Bort, and A. Muller, "Phase-sensitive single-pulse autocorrelator for ultrashort laser pulses,” Opt. Lett. 13(9), 746-748 (1988).

${ }^{4}$ P. Langlois and E. P. Ippen, "Measurement of pulse asymmetry by three-photonabsorption autocorrelation in a GaAsP photodiode," Opt. Lett. 24, 1868-1870 (1999).

${ }^{5}$ M. M. Karkhanehchi, C. J. Hamilton, and J. H. Marsh, “Autocorrelation measurements of modelocked Nd:YLF laser pulses using two-photon absorption waveguide autocorrelator," IEEE Photonics Technol. Lett. 9, 645-647 (1997).
${ }^{6}$ C. Spielmann, L. Xu, and F. Krausz, "Measurement of interferometric autocorrelations: Comment," Appl. Opt. 36, 2523-2525 (1997).

${ }^{7}$ K. N. Choi and H. F. Taylor, "Novel cross-correlation technique for characterization of subpicosecond pulses from mode-locked semiconductor lasers," Appl. Phys. Lett. 62, 1875-1877 (1993).

${ }^{8}$ Y. Takagi, T. Kobayashi, and K. Yoshihara, "Multiple- and single-shot autocorrelator based on two-photonconductivity in semiconductors," Opt. Lett. 17(9), 658-660 (1992).

${ }^{9}$ W. Rudolph, M. Sheik-Bahae, A. Bernstein, and L. F. Lester, "Femtosecond autocorrelation measurements based on two-photon photoconductivity in $\mathrm{ZnSe}$," Opt. Lett. 22, 313-315 (1997).

${ }^{10}$ R. Trebino, K. W. DeLong, D. N. Fittinghoff, J. N. Sweetser, M. A. Krumbugel, B. A. Richman, and D. J. Kane, "Measuring ultrashort laser pulses in the timefrequency domain using frequency-resolved optical gating," Rev. Sci. Instrum. 68, 3277-3295 (1997).

${ }^{11}$ D. J. Kane and R. Trebino, "Characterization of arbitrary femtosecond pulses using frequencyresolved optical gating," IEEE J. Quantum Electron. 29, 571-579 (1993).

${ }^{12}$ K. W. DeLong, R. Trebino, J. Hunter, and W. E. White, "Frequency-resolved optical gating with the use of second-harmonic generation," J. Opt. Soc. Am. B 11, 2206-2215 (1994).

${ }^{13} \mathrm{~K}$. W. DeLong and R. Trebino, "Improved ultrashort pulse-retrieval algorithm for frequency-resolved optical gating," J. Opt. Soc. Am. A 11, 2429-2437 (1994).

${ }^{14}$ J. Paye, M. Ramaswamy, J. G. Fujimoto, and E. P. Ippen, "Measurement of the amplitude and phase of ultrashort light pulses from spectrally resolved autocorrelation," Opt. Lett. 18, 1946-1948 (1993).

${ }^{15}$ D. J. Kane, A. J. Taylor, R. Trebino, and K. W. DeLong, "Singleshot measurement of the intensity and phase of a femtosecond UV laser pulse with frequency-resolved optical gating," Opt. Lett. 19, 1061-1063 (1994).

${ }^{16}$ D. J. Kane and R. Trebino, "Single-shot measurement of the intensity and phase of an arbitrary ultrashort pulse by using frequency-resolved optical gating," Opt. Lett. 18, 823-825 (1993).

${ }^{17}$ J. Peatross and A. Rundquist, "Temporal decorrelation of short laser pulses," J. Opt. Soc. Am. B 15, 216-222 (1998).

${ }^{18}$ I. Amat-Roldan, I. G. Cormack, P. Loza-Alvarez, E. J. Gualda, and D. Artigas, "Ultrashort pulse characterisation with SHG collinear-FROG," Opt. Express 12, 1169-1178 (2004).

${ }^{19}$ P. K. Bates, O. Chalus, and J. Biegert, "Ultrashort pulse characterization in the mid-infrared," Opt. Lett. 35, 1377-1379 (2010).

${ }^{20} \mathrm{R}$. Trebino and D. J. Kane, "Using phase retrieval to measure the intensity and phase of ultrashort pulses: Frequency-resolved optical gating," J. Opt. Soc. Am. A 10, 1101-1111 (1993).

${ }^{21} \mathrm{C}$. Iaconis and I. Walmsley, "Spectral phase interferometry for direct electricfield reconstruction of ultrashort optical pulses," Opt. Lett. 23, 792-794 (1998).

${ }^{22}$ P. Ośhea, M. Kimmel, X. Gu, and R. Trebino, "Highly simplified device for ultrashort-pulse measurement," Opt. Lett. 26, 932-934 (2001).

${ }^{23}$ A. S. Radunsky, I. A. Walmsley, S.-P. Gorza, and P. Wasylczyk, "Compact spectral shearing interferometer for ultrashort pulse characterization," Opt. Lett. 32, 181-183 (2007).

${ }^{24}$ V. Loriot, G. Gitzinger, and N. Forget, "Self-referenced characterization of femtosecond laser pulses by chirp scan," Opt. Express 21(21), 24879-24893 (2013). 